\title{
Muscle sphingolipids during rest and exercise: a C18:0 signature for insulin resistance in humans
}

\author{
Bryan C. Bergman ${ }^{1}$ - Joseph T. Brozinick ${ }^{2}$ - Allison Strauss ${ }^{1} \cdot$ Samantha Bacon $^{1}$ • \\ Anna Kerege ${ }^{1}$ - Hai Hoang Bui ${ }^{2}$ - Phil Sanders ${ }^{2}$ - Parker Siddall ${ }^{2}$ - Tao $\mathrm{Wei}^{2}$ • \\ Melissa K. Thomas ${ }^{2} \cdot$ Ming Shang Kuo ${ }^{2} \cdot$ Leigh Perreault $^{1}$
}

Received: 10 August 2015 / Accepted: 3 December 2015 /Published online: 6 January 2016

(C) Springer-Verlag Berlin Heidelberg 2016

\begin{abstract}
Aims/hypotheses Ceramides and other sphingolipids comprise a family of lipid molecules that accumulate in skeletal muscle and promote insulin resistance. Chronic endurance exercise training decreases muscle ceramides and other sphingolipids, but less is known about the effects of a single bout of exercise.

Methods We measured basal relationships and the effect of acute exercise $\left(1.5 \mathrm{~h}\right.$ at $\left.50 \% \dot{V}_{2 \max }\right)$ and recovery on muscle sphingolipid content in obese volunteers, endurance trained athletes and individuals with type 2 diabetes.

Results Muscle C18:0 ceramide $(p=0.029)$, dihydroceramide $(p=0.06)$ and glucosylceramide $(p=0.03)$ species were inversely related to insulin sensitivity without differences in total ceramide, dihydroceramide, and glucosylceramide concentration. Muscle C18:0 dihydroceramide correlated with markers of muscle inflammation $(p=0.04)$. Transcription of genes encoding sphingolipid synthesis enzymes was higher in athletes, suggesting an increased capacity for sphingolipid synthesis. The total concentration of muscle ceramides and sphingolipids increased during exercise and then decreased after recovery, during which time ceramide levels reduced to significantly below basal levels.
\end{abstract}

Electronic supplementary material The online version of this article (doi:10.1007/s00125-015-3850-y) contains peer-reviewed but unedited supplementary material, which is available to authorised users.

Bryan C. Bergman

Bryan.Bergman@ucdenver.edu

1 Division of Endocrinology, Metabolism, and Diabetes, University of Colorado Anschutz Medical Campus, PO Box 6511, MS 8106, Aurora, CO 80045, USA

2 Eli Lilly and Company, Indianapolis, IN, USA
Conclusions/interpretation These data suggest ceramide and other sphingolipids containing stearate (18:0) are uniquely related to insulin resistance in skeletal muscle. Recovery from an exercise bout decreased muscle ceramide concentration; this may represent a mechanism promoting the insulinsensitising effects of acute exercise.

Keywords Athlete's paradox $\cdot$ Insulin sensitivity $\cdot$ Lipid composition
Abbreviations
CerS1 Ceramide synthase 1
CTRC Clinical Translational Research Center
ERK1/2 Extracellular signal-related kinase 1/2
GM3 ( $N$-Acetylneuraminyl)-D-galactosyl-D- glucosylceramide
IKK $\alpha$ Inhibitor of nuclear factor kappa-B kinase sub-
JNK $\quad$ unit alpha
MAP4K4 Mitogen-activated protein kinase kinase kinase kinase 4
PP2A Protein phosphatase 2A
TLR4 Toll-like receptor 4
$\dot{V} \mathrm{O}_{2 \max } \quad$ Maximal oxygen consumption

\section{Introduction}

As the epidemic of obesity and type 2 diabetes persists, the need for new therapeutic targets to enhance muscle insulin sensitivity has become dire. The accumulation of muscle lipids are related to insulin resistance, and diacylglycerols, ceramides and other sphingolipids, and long chain acyl-CoA are thought to play direct roles in reducing insulin sensitivity, 
thus making them prime drug targets [1-3]. Ceramides and other sphingolipids are involved in the structure of membrane lipids and are important signalling molecules that influence apoptosis, inflammation and insulin sensitivity $[1,3]$. They comprise a complex family of related molecules, as exemplified by the fact that ceramide can be phosphorylated to ceramide-1-phosphate, glycosylated to glucosylceramide (which is a precursor to many other species) or converted to sphingosine, which can be further phosphorylated [3]. Although it has been established that high concentrations of ceramide in skeletal muscle are related to insulin resistance [4-9], the exact role of unique ceramide and other sphingolipid species in muscle insulin resistance is unknown.

Chronic endurance exercise training is known to decrease muscle ceramide concentration [10-12], but the influence of an acute bout of exercise is less well understood. For example, a single bout of endurance exercise has been shown to enhance insulin sensitivity [13] but, paradoxically, muscle ceramide concentration is increased immediately after exercise in humans [14]. This observation directly contrasts with studies in rodents, in which ceramides are reduced after exercise [15]. Finally, it is not known whether exercise-induced changes in muscle ceramides and other sphingolipids are altered in insulin-resistant individuals.

We sought to perform the most complete evaluation to date of the molecular species of ceramides and other sphingolipids in the muscle and serum in a population of individuals with a broad range of insulin sensitivities. This allowed us to determine which of these species were related to insulin resistance and how they changed immediately following an acute exercise bout and after $2 \mathrm{~h}$ of recovery. Understanding which of the muscle lipids related to insulin resistance change with acute exercise may provide insight into potential therapeutic targets for insulin sensitisation.

\section{Methods}

Participants In all, 14 obese sedentary individuals, 15 type 2 diabetic patients and 15 endurance trained athletes were recruited for this study. Serum sphingolipid data from this study was published previously [16]. All participants gave written informed consent and were excluded if they had a BMI of $<20$ or $>25 \mathrm{~kg} / \mathrm{m}^{2}$ (for athletes) or of $<28$ or $>40 \mathrm{~kg} /$ $\mathrm{m}^{2}$ (for obese and type 2 diabetic individuals), fasting triacylglycerol levels of $>1.7 \mathrm{mmol} / \mathrm{l}$, or liver, kidney, thyroid or lung disease. Sedentary participants had been engaged in planned physical activity for $<2 \mathrm{~h} /$ week. Endurance trained participants were competitive cyclists, triathletes and runners with average lactate thresholds of $78 \pm 1.4 \%$ maximal oxygen consumption $\left(\dot{V} \mathrm{O}_{2 \max }\right)$ who had been training for an average of $12.3 \pm 0.8 \mathrm{~h} /$ week over the previous $9.7 \pm 2.1$ years for the purpose of competition. Individuals with type 2 diabetes were excluded from the study if they used insulin and/or thiazolidinediones. All other medications were permissible but were washed out for 2 weeks prior to metabolic testing. One individual was on beta-blockers, two were on ACE inhibitors, five were on statins, three were on sulfonylureas and six were on metformin. Obese participants and athletes were not taking medication. Participants were weight stable in the 6 months prior to the study. This study was approved by the Colorado Multiple Institution Review Board at the University of Colorado Denver.

Preliminary testing Following a $12 \mathrm{~h}$ overnight fast, participants reported to the Clinical Translational Research Center (CTRC) for screening, which consisted of a health and physical examination followed by a fasting blood draw. Body composition was determined using dual energy x-ray absorptiometry (DEXA) (DPX-IQ, Lunar Corporation, Madison, WI, USA).

Insulin sensitivity was then determined via an IVGTT using standard methods (see electronic supplementary material [ESM] Methods and Results for further details) [17].

Diet and exercise control All participants were given a prescribed diet for 3 days prior to admission to the CTRC. The daily caloric requirement was estimated as previously described [18]. Composition of this diet was 55\% carbohydrate, $30 \%$ fat and $15 \%$ protein. The composition of the fat content of the diet was controlled: saturated, monounsaturated and polyunsaturated fat was present in a 1:1:1 ratio. Participants were asked to refrain from any planned physical activity for $48 \mathrm{~h}$ before the metabolic study.

Metabolic study After a $12 \mathrm{~h}$ overnight fast, an antecubital vein in one arm was cannulated for isotope infusion and a retrograde dorsal hand vein in the contralateral side was catheterised for blood sampling via the heated hand technique. Data from $\left[\mathrm{U}-{ }^{13} \mathrm{C}\right]$ palmitate and $\left[6,6-{ }^{2} \mathrm{H}_{2}\right]$ glucose infusions will be reported separately. After resting for $3.5 \mathrm{~h}$, blood sampling was performed every $10 \mathrm{~min}$ for $30 \mathrm{~min}$ for metabolic and hormone analysis. A percutaneous needle biopsy $(\sim 150 \mathrm{mg})$ was then taken from midway between the greater trochanter of the femur and the patella. The skeletal muscle sample was immediately flash frozen in liquid nitrogen and stored at $-80^{\circ} \mathrm{C}$ until analysis. Extramuscular fat was dissected from samples on ice as previously described [19].

After the resting muscle biopsy, participants exercised on a cycle ergometer for $1.5 \mathrm{~h}$ at $50 \%$ of $\dot{V} \mathrm{O}_{2 \max }$. Exercise intensity was determined using indirect calorimetry and the exercise workload was adjusted so that the relative intensity was maintained throughout exercise. Blood sampling was performed during the last $30 \mathrm{~min}$ of exercise. A muscle biopsy was taken within $1 \mathrm{~min}$ after stopping exercise from the same site as 
previously used, but with the needle at a more proximal angle. Participants then remained supine for $2 \mathrm{~h}$ of recovery. Blood sampling was performed during the last $30 \mathrm{~min}$ of recovery, followed by muscle biopsy from the contralateral leg. Participants fasted throughout rest, exercise and recovery: only water consumption was allowed.

Substrate and hormone analyses Standard assays were used for substrate and hormone analysis (see ESM Methods and Results for further details).

Lipidomics analysis Liquid chromatography/electrospray ionisation/triple quadrupole MS (LC/ESI/MS/MS) analysis of ceramide and sphingolipids was performed as previously described (see ESM Methods and Results for further details) [16].

Gene chip analysis For RNA extraction, skeletal muscle biopsies were lysed in Trizol (Invitrogen, Life Technologies, Grand Island, NY, USA) using a FastPrep instrument and lysing matrix D tubes (MP Biomedicals, Santa Ana, CA, USA). RNA was then isolated using chloroform extraction and ethanol precipitation, further purified using RNeasy columns (Qiagen, Valencia, CA, USA), DNase treated and eluted with RNase-free water. Purified RNA was used for Affymetrix (Santa Clara, CA, USA) gene chip analysis according to the manufacturer's instructions [20].

Western blotting Inflammatory marker levels and basal insulin signalling were determined by western blotting (see ESM Methods and Results for further details).

Statistical analysis Data are presented as the mean \pm SEM. Differences in normally distributed basal data among groups were analysed by one-way ANOVA (Version 22, SPSS, IBM, Armonk, NY, USA). Non-normally distributed data were log-transformed (base 10) prior to analysis. When significant differences were detected, individual means were compared using Student's $t$ tests to determine differences among groups. Differences in total sphingolipid species after exercise and recovery were determined using repeated measures ANOVA. When a significant temporal effect was found, paired $t$ tests were performed to examine changes in individual species by group. An $\alpha$ level of 0.05 was considered statistically significant. Relationships between measurements were determined using Pearson's $r$. Gene transcription data for each probe set were analysed using repeated measures ANOVA with a compound symmetric covariance structure and repeated measures based on the activity periods. Linear contrasts within the repeated measures analysis were used to evaluate comparisons among groups and over time for each probe set. Differences in gene transcription during rest, exercise and recovery were made using paired comparisons and corrected for three comparisons using Bonferroni, resulting in statistical significance being set at a $p$ value of $<0.017$.

\section{Results}

Demographics As previously reported, there were no significant differences in age (in years: obese, $39.7 \pm 1.6$; type 2 diabetes, $42.5 \pm 1.1$; athletes, $41.3 \pm 0.86$ ) or sex (men/women: obese, 9/5; type 2 diabetes, 11/4; athletes, 11/4) among groups [16]. As expected, obese and type 2 diabetic individuals had greater BMI (in $\mathrm{kg} / \mathrm{m}^{2}$ : obese, $33.2 \pm 0.8$; type 2 diabetes, 29.9 \pm 2.6 ; athletes, $24.2 \pm 0.6$ ) and percentage body fat (in \%: obese, $33.2 \pm 2.0$; type 2 diabetes, $27.2 \pm 4.0$; athletes, 17.0 $\pm 1.6)$ compared with athletes $(p<0.0001)$. Athletes had roughly twice the $\dot{V} \mathrm{O}_{2 \max }$ of obese and type 2 diabetic individuals (in $\mathrm{ml} \mathrm{kg}^{-1} \mathrm{~min}^{-1}$ : obese, $23.8 \pm 2.5$; type 2 diabetes, $18.7 \pm 2.7$; athletes, $47.8 \pm 3.8 ; p<0.0001$ ). The $\mathrm{HbA}_{1 \mathrm{c}}$ (in \%: obese, $5.4 \pm 0.1$; type 2 diabetes, $7.9 \pm 0.6$; athletes, $5.4 \pm 0.1$; in $\mathrm{mmol} / \mathrm{mol}$ : obese, $35.4 \pm 0.8$; type 2 diabetes, $60.5 \pm 7.0$; athletes, $35.9 \pm 0.7$ ) and $2 \mathrm{~h}$ glucose (in mmol/l: obese, 4.8 \pm 0.3 ; type 2 diabetes, $14.9 \pm 1.0$; athletes, $4.2 \pm 0.4$ ) were significantly higher in type 2 diabetic patients than in the other two groups $(p<0.0001)$. Insulin sensitivity was significantly greater in athletes $\left(9.4 \pm 1.0 \mathrm{mU} \mathrm{l}^{-1} \mathrm{~min}^{-1}\right.$, [convert to SI units by multiplying by 0.167$]$ than in obese and type 2 diabetic individuals (in $\mathrm{mU}^{-1} \mathrm{~min}^{-1}$ : obese, $2.93 \pm 0.2$; type 2 diabetes, $2.0 \pm 0.3 ; p<0.0001)$.

Serum palmitate $(\mathrm{C} 16: 0)$ and stearate $(\mathrm{C} 18: 0)$ concentrations were significantly higher in type 2 diabetic patients than in the other two groups at baseline $(p=0.02$ and $p=0.006$, respectively; Table 1). Total NEFA concentration significantly increased from rest to exercise $(p<0.0001)$ and continued to increase during recovery $(p<0.0001$; Table 2$)$. Resting glucose and insulin concentrations were significant greater in type 2 diabetic patients than in the other two groups. During exercise, most metabolites (other than glucose and insulin) increased significantly compared with rest. During recovery, NEFA continued to increase, while most metabolites returned to near basal levels. Of note, TNF- $\alpha$ significantly increased during exercise $(p=0.005)$ and returned to basal levels during recovery. When evaluated by group, the increase in TNF- $\alpha$ during exercise only reached statistical significance in type 2 diabetic patients $(p=0.04)$.

Muscle ceramides and sphingolipids We found no significant between-group differences in skeletal muscle sphingosine or sphingosine-1-phosphate at rest (Fig. 1a). In all groups combined, exercise induced a significant increase in sphingosine $(p=0.03)$ and sphingosine-1-phosphate $(p<0.0001)$ concentration, with sphingosine tending to be reduced during recovery compared with at rest ( $p=0.058$; Fig. $1 \mathrm{~b})$. The resting 
Table 1 Serum NEFA abundance at rest by group

\begin{tabular}{lccc}
\hline NEFA (AU) & $\begin{array}{l}\text { Obese individuals } \\
(n=14)\end{array}$ & $\begin{array}{l}\text { Type 2 diabetic patients } \\
(n=15)\end{array}$ & Athletes $(n=14)$ \\
\hline 14:0 myristic acid & $0.14 \pm 0.009$ & $0.14 \pm 0.006$ & $0.14 \pm 0.009$ \\
16:0 palmitic acid & $2.15 \pm 0.17$ & $2.62 \pm 0.15^{*}$ & $1.98 \pm 0.12$ \\
16:1 palmitoleic acid & $0.28 \pm 0.03$ & $0.27 \pm 0.03$ & $0.25 \pm 0.02$ \\
18:0 stearic acid & $2.03 \pm 0.16$ & $2.85 \pm 0.25^{*}$ & $2.14 \pm 0.11$ \\
18:1 oleic acid & $4.78 \pm 0.61$ & $5.26 \pm 0.36$ & $4.58 \pm 0.44$ \\
18:2 linoleic acid & $2.54 \pm 0.29$ & $2.55 \pm 0.21$ & $2.36 \pm 0.23$ \\
18:3 linolenic acid & $0.17 \pm 0.02$ & $0.16 \pm 0.01$ & $0.15 \pm 0.02$ \\
20:0 arachidic acid & $0.02 \pm 0.002$ & $0.04 \pm 0.004^{\star}$ & $0.03 \pm 0.001$ \\
20:1 eicosenoic acid & $0.05 \pm 0.007$ & $0.05 \pm 0.004$ & $0.05 \pm 0.004$ \\
20:4 arachidonic acid & $0.46 \pm 0.09$ & $0.35 \pm 0.05$ & $0.32 \pm 0.06$ \\
20:5 eicosapentaenoic acid & $0.09 \pm 0.03^{*}$ & $0.03 \pm 0.007$ & $0.04 \pm 0.008$ \\
20:2 eicosadienoic acid & $0.04 \pm 0.005$ & $0.05 \pm 0.003$ & $0.04 \pm 0.003$ \\
22:0 behenic acid & $0.007 \pm 0.0005$ & $0.007 \pm 0.0006$ & $0.007 \pm 0.0004$ \\
22:1 erucic acid & $0.003 \pm 0.0002^{*}$ & $0.004 \pm 0.0003^{*}$ & $0.005 \pm 0.0004$ \\
22:6 docosahexaenoic acid & $0.14 \pm 0.03$ & $0.08 \pm 0.009$ & $0.15 \pm 0.04$ \\
24:0 lignoceric acid & $0.01 \pm 0.001$ & $0.01 \pm 0.001$ & $0.01 \pm 0.001$ \\
\hline
\end{tabular}

*Significantly different than in athletes

${ }^{\dagger}$ Significantly different than in type 2 diabetic patients

${ }^{\star}$ Significantly different than in obese individuals

$p<0.05$ for all concentration of muscle sphingosine and sphingosine-1phosphate were not significantly related to insulin sensitivity.

The total muscle ceramide concentration was not related to BMI or to insulin resistance and was not different among groups at rest (Fig. 2a); however, individual ceramide species differed. The muscle C18:0 ceramide concentration was significantly higher in type 2 diabetic patients than in athletes $(p=0.007)$; it was positively associated with BMI $(p=0.002)$ and inversely related to insulin sensitivity $(p=0.029$; Fig. 2e). The total muscle ceramide concentration was significantly increased by acute exercise $(p=0.05)$ and tended to be lower after recovery than at rest $(p=0.055$; Fig. 2b). These findings were largely driven by changes in type 2 diabetic patients (Fig. 2c, d). C18:0 ceramide was significantly greater in obese and type 2 diabetic individuals than in athletes after acute exercise (ng/mg wet weight: obese, $1.5 \pm 0.20$; type 2 diabetes, $2.1 \pm 0.18$; athletes, $0.9 \pm 0.10$; $p \leq 0.03)$. This difference persisted during recovery between type 2 diabetic patients and athletes ( $\mathrm{ng} / \mathrm{mg}$ wet weight: type 2 diabetes, $1.3 \pm 0.14$; athletes, $0.9 \pm 0.12 ; p=0.02$ ).

The total muscle dihydroceramide concentration was not different among groups at baseline and was not related to BMI or insulin resistance, although there was a significantly higher C18:0 dihydroceramide content in obese participants compared with athletes $(p=0.007$; Fig. 3a). There was a positive relationship between $\mathrm{C} 18: 0$ dihydroceramide and BMI $(p=0.04)$ and an inverse relationship between C18:0 dihydroceramide and insulin sensitivity that approached significance $(p=0.06)$. The dihydroceramide concentration was significantly increased by exercise $(p=0.03)$ and decreased during recovery to basal values (Fig. 3b). Increased C24:0 dihydroceramide in obese participants during exercise helped explain the overall increase during exercise compared with at rest (Fig. 3c).

The resting concentration of total muscle glucosylceramide was not significantly different among groups (Fig. 4a). The C18:0 glucosylceramide content was significantly higher in obese and type 2 diabetic individuals than in athletes $(p=0.04)$, and was inversely related to BMI $(p=0.009)$ and insulin sensitivity ( $p=0.03$; Fig. $4 \mathrm{e})$. Exercise increased the muscle glucosylceramide concentration $(p=0.02)$, which decreased during recovery to values that tended to be lower than at rest (Fig. 4b; $p=0.06$ ). The increase in $\mathrm{C} 16: 0$ glucosylceramide in obese and type 2 diabetic individuals and the increase in C24:0 and C24:1 in athletes accounted for the overall increase in muscle ceramide with exercise (Fig. 4c). Obese and type 2 diabetic individuals tended to have decreased total glucosylceramide during recovery, in contrast to athletes (Fig. 4d). The difference in C18:0 glucosylceramide levels between type 2 diabetic patients and athletes was maintained during both exercise $(p=0.0008)$ and recovery $(p=0.02)$.

Muscle GM3 ganglioside [( $N$-acetylneuraminyl)-Dgalactosyl-D-glucosylceramide] concentration was not 
Table 2 Plasma and serum concentrations by group

\begin{tabular}{|c|c|c|c|}
\hline Variable & $\begin{array}{l}\text { Obese individuals } \\
(n=14)\end{array}$ & $\begin{array}{l}\text { Type } 2 \text { diabetic patients } \\
(n=15)\end{array}$ & Athletes $(n=15)$ \\
\hline \multicolumn{4}{|l|}{ Rest } \\
\hline Glucose (mmol/l) & $4.9 \pm 0.1$ & $7.6 \pm 0.9^{* \ddagger}$ & $4.7 \pm 0.1$ \\
\hline NEFA (mmol/l) & $0.628 \pm 0.04$ & $0.663 \pm 0.05$ & $0.632 \pm 0.04$ \\
\hline Triacylglycerol (mmol/l) & $1.6 \pm 0.2^{*}$ & $1.5 \pm 0.2 *$ & $0.82 \pm 0.1$ \\
\hline Glycerol (mmol/1) & $0.09 \pm 0.01$ & $0.10 \pm 0.01$ & $0.09 \pm 0.01$ \\
\hline Lactate $(\mathrm{mmol} / \mathrm{l})$ & $0.67 \pm 0.10$ & $0.76 \pm 0.08$ & $0.64 \pm 0.07$ \\
\hline Insulin (pmol/l) & $45.1 \pm 6.3$ & $106.3 \pm 18.1^{* *}$ & $24.3 \pm 6.3$ \\
\hline Glucagon (ng/l) & $66.7 \pm 3.6^{*}$ & $75.3 \pm 4.9^{*}$ & $53.7 \pm 2.5$ \\
\hline Epinephrine (pmol/l) & $129.4 \pm 11.5$ & $139.2 \pm 12.6$ & $144.7 \pm 10.4$ \\
\hline Noradrenaline (pmol/l) & $1,419 \pm 201$ & $1,365 \pm 112$ & $1,371 \pm 142$ \\
\hline $\mathrm{TNF}-\alpha(\mathrm{ng} / \mathrm{l})$ & $7.1 \pm 0.3$ & $6.4 \pm 0.3$ & $7.1 \pm 0.3$ \\
\hline \multicolumn{4}{|l|}{ Exercise } \\
\hline Glucose (mmol/l) & $4.7 \pm 0.1^{\S}$ & $6.8 \pm 0.7^{*}$ & $4.8 \pm 0.1$ \\
\hline NEFA (mmol/l) & $0.812 \pm 0.06^{\S}$ & $0.773 \pm 0.07^{\S}$ & $0.761 \pm 0.06^{\S}$ \\
\hline Triacylglycerol (mmol/l) & $1.7 \pm 0.2 * \S$ & $1.7 \pm 0.2 * \S$ & $1.0 \pm 0.1^{\S}$ \\
\hline Glycerol (mmol/l) & $0.21 \pm 0.02^{\S}$ & $0.25 \pm 0.03^{\S}$ & $0.34 \pm 0.03^{\dagger+\S}$ \\
\hline Lactate $(\mathrm{mmol} / \mathrm{l})$ & $1.73 \pm 0.3^{\S}$ & $2.5 \pm 0.3 * \nsubseteq \S$ & $1.1 \pm 0.1^{\S}$ \\
\hline Insulin $(\mathrm{pmol} / \mathrm{l})$ & $32.6 \pm 4.9^{\S}$ & $97.9 \pm 18.1^{* *}$ & $21.5 \pm 1.4$ \\
\hline Glucagon (ng/l) & $86.8 \pm 7.5^{*} \S$ & $110.3 \pm 12.4^{* \S}$ & $56.8 \pm 4.4$ \\
\hline Epinephrine (pmol/l) & $385.4 \pm 37^{\S}$ & $517 \pm 80.8^{\S}$ & $522 \pm 51^{\S}$ \\
\hline Noradrenaline (pmol/l) & $3,913 \pm 497^{\S}$ & $4,528 \pm 325^{\S}$ & $7,330 \pm 869^{\dagger+\S}$ \\
\hline $\mathrm{TNF}-\alpha(\mathrm{ng} / \mathrm{l})$ & $7.4 \pm 0.3$ & $7.1 \pm 0.5^{\S}$ & $7.4 \pm 0.4$ \\
\hline \multicolumn{4}{|l|}{ Recovery } \\
\hline Glucose (mmol/l) & $4.7 \pm 0.1^{\S}$ & $6.3 \pm 0.7 * \pm \S$ & $4.5 \pm 0.1^{\S}$ \\
\hline NEFA (mmol/l) & $1.01 \pm 0.06^{8 \mp}$ & $1.09 \pm 0.07^{\S \uparrow}$ & $1.01 \pm 0.05^{\S \uparrow}$ \\
\hline Triacylglycerol (mmol/l) & $1.4 \pm 0.2 * 8 \pi$ & $1.5 \pm 0.2 * \pi$ & $0.8 \pm 0.1^{\llbracket}$ \\
\hline Glycerol (mmol/l) & $0.12 \pm 0.01^{\text {s๘ }}$ & $0.14 \pm 0.01^{\S \Psi}$ & $0.13 \pm 0.01^{\text {s๘ }}$ \\
\hline Lactate $(\mathrm{mmol} / \mathrm{l})$ & $0.77 \pm 0.1^{\llbracket}$ & $1.05 \pm 0.2^{\S \uparrow}$ & $0.86 \pm 0.1^{\S \uparrow}$ \\
\hline Insulin $(\mathrm{pmol} / \mathrm{l})$ & $36.8 \pm 5.6^{\S}$ & $83.3 \pm 14.6^{* \star}$ & $25 \pm 2.1$ \\
\hline Glucagon (ng/l) & $70.9 \pm 6.4 * \pi$ & $85.0 \pm 5.6^{* \S \varpi}$ & $53.5 \pm 2.7 *$ \\
\hline Epinephrine (pmol/l) & $130 \pm 9.3^{\pi}$ & $186 \pm 22 *$ & $137 \pm 8.7^{\natural}$ \\
\hline Noradrenaline (pmol/l) & $1,501 \pm 219^{\natural}$ & $1,903 \pm 195^{\S \varpi}$ & $2,258 \pm 302^{8 ๘}$ \\
\hline TNF- $\alpha(n g / 1)$ & $7.1 \pm 0.4$ & $6.2 \pm 0.3^{\pi}$ & $6.8 \pm 0.3$ \\
\hline
\end{tabular}

* Significantly different than in athletes

${ }^{\dagger}$ Significantly different than in type 2 diabetic patients

* Significantly different than in obese individuals

$\S$ Significantly different than at rest

" Significantly different than during exercise

$p<0.05$ for all different among groups at rest; however, athletes had a significantly greater C20:0 $(p=0.006)$ and C22:0 $(p=0.002)$ content compared with obese and type 2 diabetic individuals (Fig. 5). The C20:0 and $C 22: 0$ ganglioside species were inversely related to BMI $(p=0.0001$ and $<0.0001$, respectively) and positively related to insulin sensitivity ( $p=0.0004$ and $p=0.002$, respectively).
Relationships between serum NEFA and muscle ceramides Total NEFA content correlated positively with total muscle sphingosine $(r=0.42, p=0.04)$, sphingosine-1phosphate $(r=0.44, p=0.03)$, ceramides $(r=0.51, p=0.007)$ and glucosylceramides $(r=0.45, p=0.02)$. Total saturated serum fatty acid content also significantly correlated with muscle total ceramides $(r=0.47, p=0.015)$, sphingosine 
Fig. 1 (a) Muscle sphingosine and sphingosine-1-phosphate (sphingosine-1-P) concentrations in the different groups at rest and (b) combined values for all groups at rest and during exercise and recovery. Percentage changes (c) from rest to exercise and (d) from rest to recovery. All values are means \pm SEM. Dashed line, sphingosine; solid line, sphingosine-1-phosphate; white bars, obese individuals; light grey bars, type 2 diabetic patients; dark grey bars, athletes. ${ }^{\S}$ Significantly different from at rest; I significantly different from during exercise $(p<0.05$ for all) $\mathbf{a}$

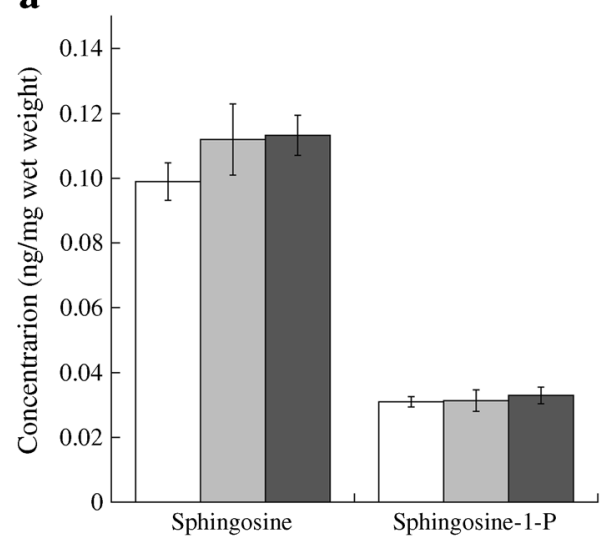

c

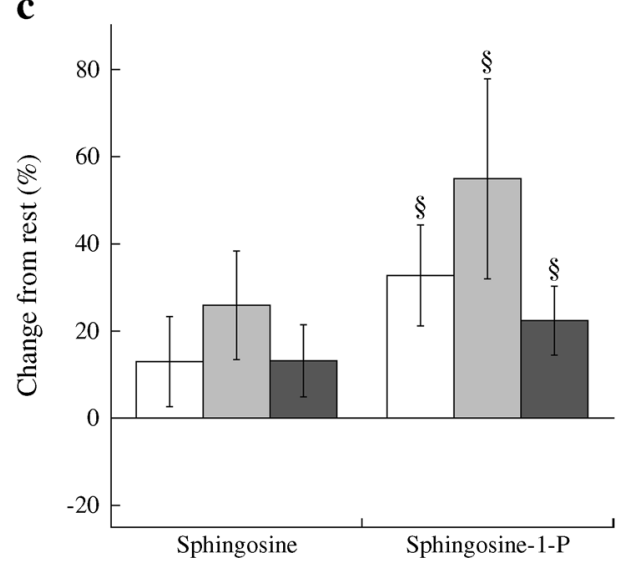

b

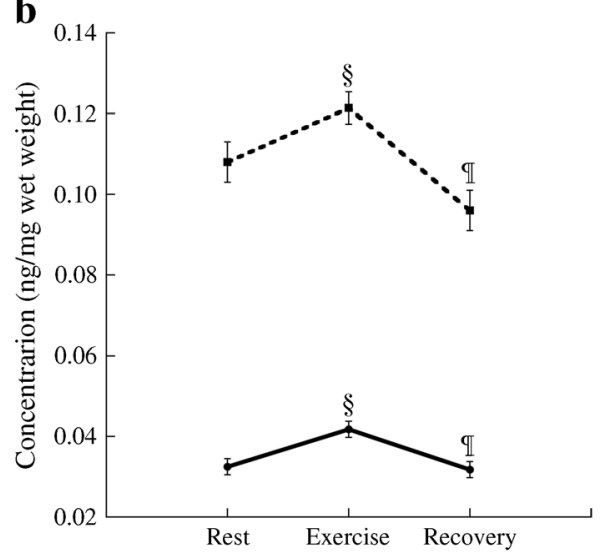

d

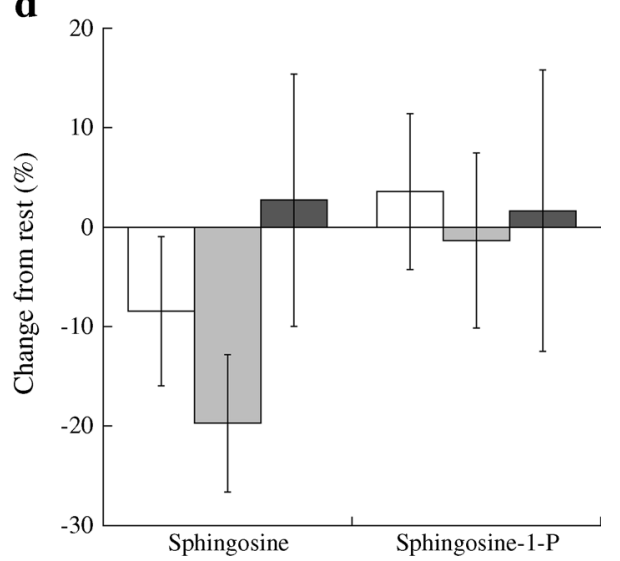

( $r=0.45, p=0.02)$, sphingosine-1 -phosphate $(r=0.45$, $p=0.018)$ and glucosylceramides $(r=0.51, p=0.005)$.

Gene transcription Basal mRNA expression of several genes involved in sphingolipid synthesis, including serine palmitoyltransferase 2, as well as sphingomyelinase 1 and 3, were lower in obese and type 2 diabetic individuals than in athletes (Fig. 6a). mRNA expression of genes involved in ceramide clearance were also lower in obese and type 2 diabetic individuals, including alkaline ceramidase 3 and sphingosine kinase 2 , while sphingomyelin synthase 2 was higher in obese and type 2 diabetic individuals. During exercise and recovery, there were no changes in mRNA expression of genes involved in ceramide production, while mRNA expression of several genes responsible for ceramide clearance were significantly increased (Fig. 6b, c). Basal mRNA expression of several genes implicated in mitophagy is shown in ESM Fig. 1.

Muscle protein expression Resting muscle c-Jun N-terminal kinase (JNK) Thr183 and Thr185 phosphorylation was significantly higher in obese and type 2 diabetic individuals than in athletes (Fig. 7a), and inversely correlated with insulin sensitivity $(r=-0.43, p=0.01)$. No significant differences in inhibitor of nuclear factor kappa-B kinase subunit alpha $(\mathrm{IKK} \alpha)$ phosphorylation were found among groups, as previously described [16], nor in extracellular-signal-regulated kinases 1 and 2 (ERK1/2) phosphorylation, or in Toll-like receptor 4 (TLR4) or mitogen-activated protein kinase kinase kinase kinase 4 (MAP4K4) content (Fig. 7b-d). During exercise, only JNK Thr183/Thr185 phosphorylation was significantly increased. After recovery, there were no significant differences in any of the proteins measured compared with at rest. Basal protein kinase B (Akt) Ser473 phosphorylation was higher in athletes than in the other two groups, with no differences found between exercise and recovery (ESM Fig. 2). There were no differences in basal TBC1 domain family member 4 (AS160) Thr642 phosphorylation among groups at any time. The muscle C18:0 dihydroceramide content had a significant positive relationship with phosphorylated JNK $(p=0.04)$ and an inverse relationship with $\mathrm{C} 22: 0$ gangliosides $(p=0.007)$.

\section{Discussion}

The relationship of individual muscle ceramides and other sphingolipid species with insulin resistance in 
Fig. 2 (a) Concentrations of muscle ceramide species in the different groups at rest and (b) combined values for all groups at rest and during exercise and recovery. Percentage changes (c) from rest to exercise and (d) from rest to recovery. White bars, obese individuals; light grey bars, type 2 diabetic patients; dark grey bars, athletes. All values are means \pm SEM. * Significantly different from in athletes; ${ }^{\dagger}$ significantly different from in type 2 diabetic patients; " significantly different from in obese individuals; $\S_{\text {significantly different from at }}$ rest; "significantly different from during exercise ( $p<0.05$ for all).

(e) Scatterplot showing the relationship between C18:0 ceramide concentration and insulin sensitivity. Circles, obese individuals; triangles, type 2 diabetic patients; squares, athletes $(r=-0.37, p=0.029)$. Cer, ceramide $\mathbf{a}$

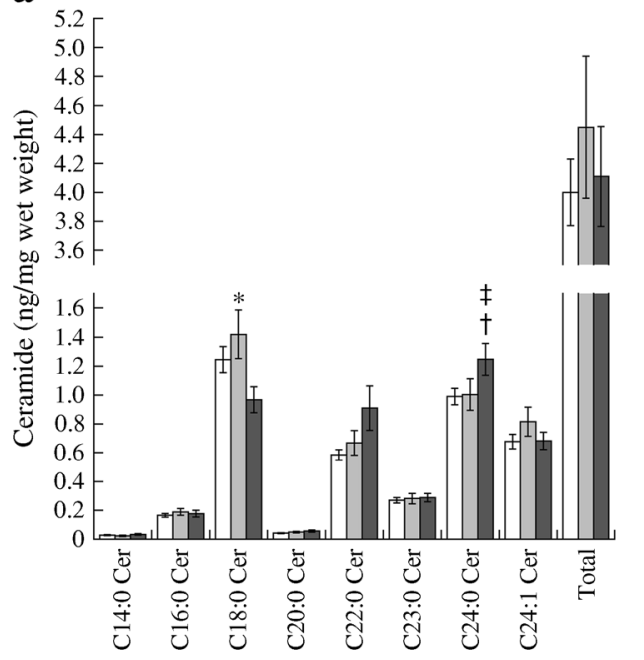

c

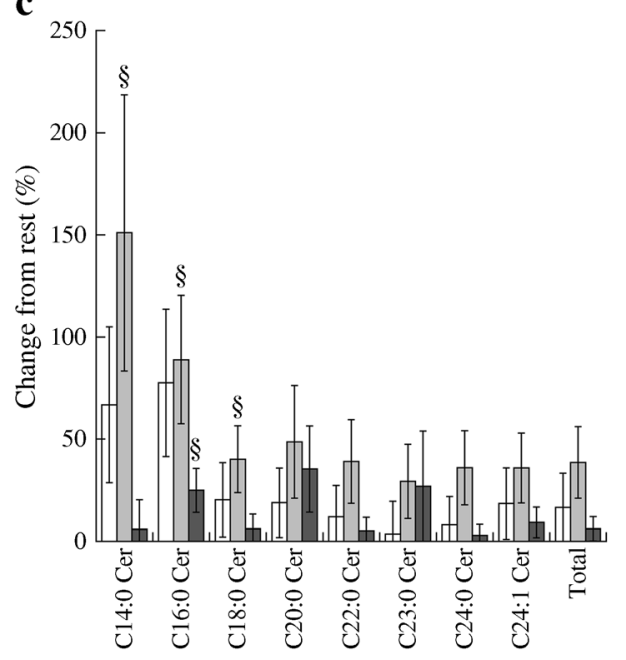

e

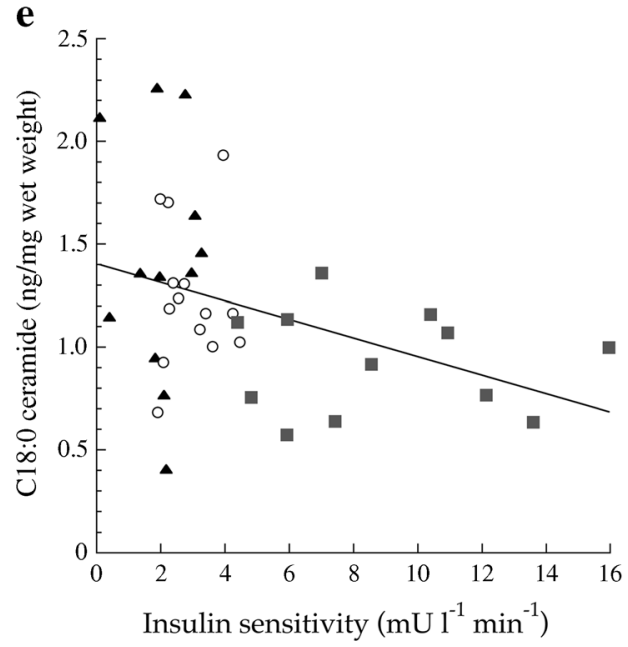

b

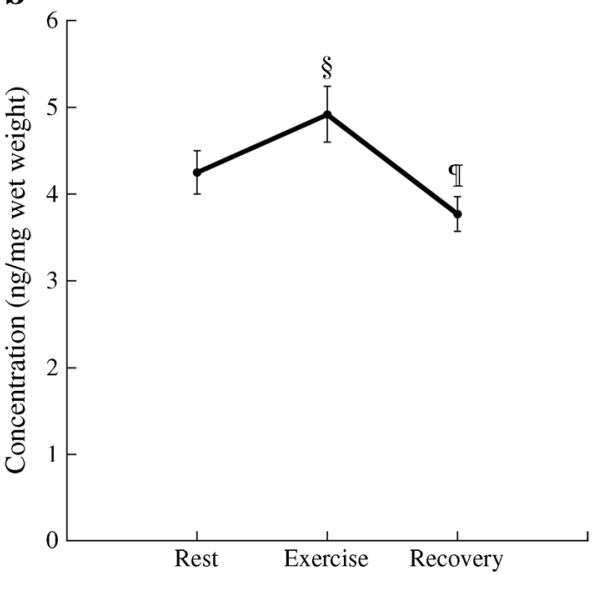

d

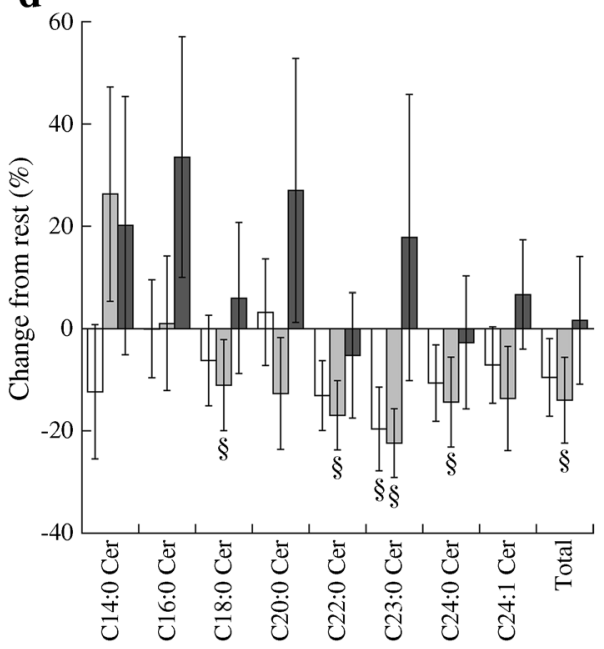

humans and the influence of acute exercise and recovery on these lipids is not well understood. A major finding of the current study is that content of muscle C18:0 ceramide, dihydroceramide and glucosylceramide species are greater in obesity and in insulin resistance. Total muscle ceramide and glucosylceramide content can be decreased after recovery from acute exercise. These findings expand our knowledge of the insulin-sensitising 
Fig. 3 (a) Concentrations of muscle dihydroceramide species in the different groups at rest and (b) combined values for all groups at rest and during exercise and recovery. Percentage changes (c) from rest to exercise and (d) from rest to recovery. All values are means \pm SEM. White bars, obese individuals; light grey bars, type 2 diabetic patients; dark grey bars, athletes. ${ }^{*}$ Significantly different from in athletes; $\S_{\text {significantly different from at }}$ rest; " significantly different from during exercise $(p<0.05$ for all). DHCer, dihydroceramide
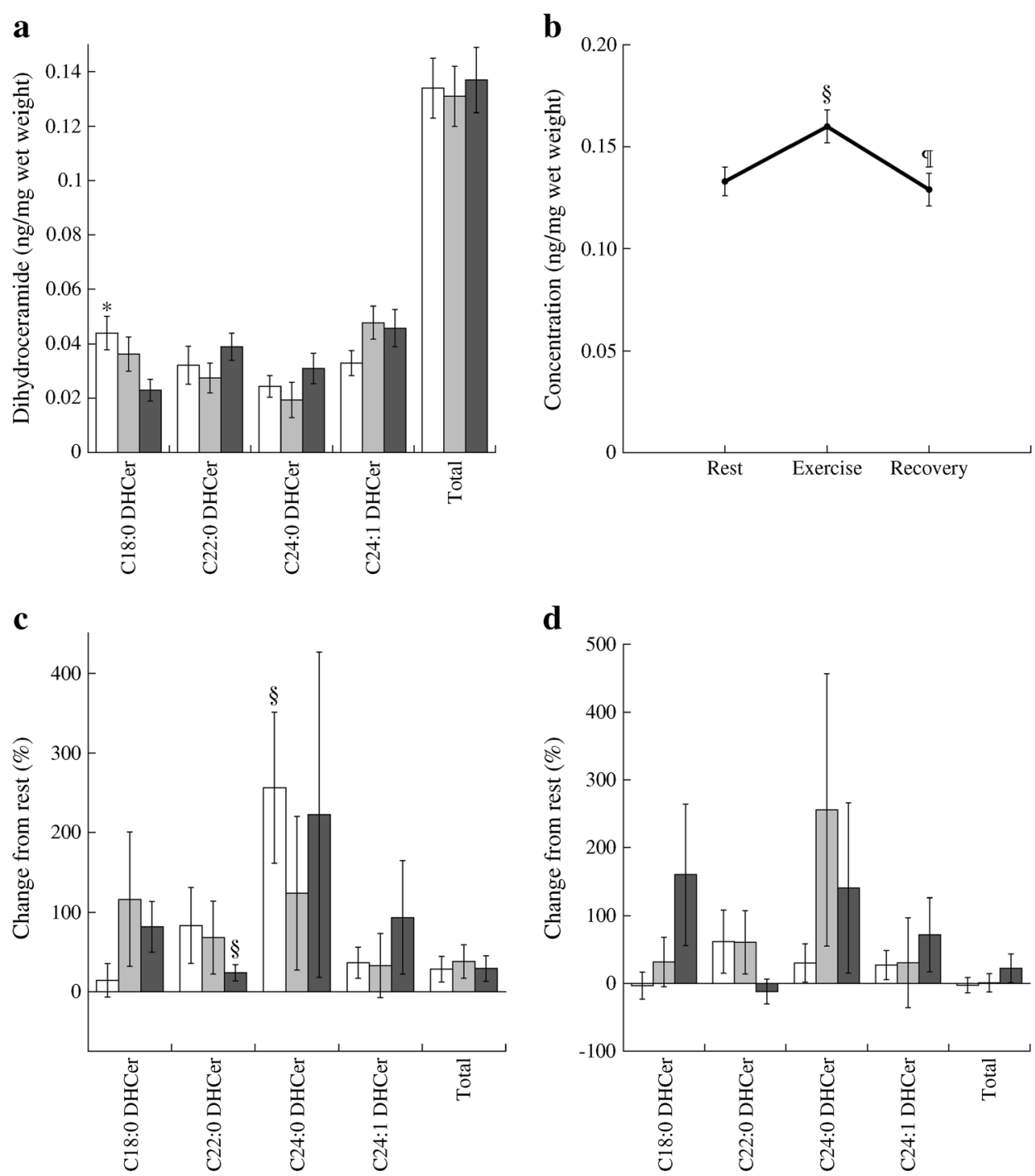

mechanisms of acute exercise and provide new targets for drug development.

Muscle ceramide accumulation is thought to be one mechanism promoting insulin resistance in humans [4-6, 21]. However, ceramide accumulation can be dissociated from insulin sensitivity, indicating that total concentration may not the only variable influencing insulin action [22-24]. It is possible that only specific ceramide or other sphingolipid species promote insulin resistance, as has been shown for diacylglycerol [18]. However, the relationship between specific ceramide species and insulin sensitivity is unclear $[12,25]$. Our data suggest that C18:0 ceramide and glucosylceramide species are uniquely related to obesity and insulin resistance in human skeletal muscle, which parallels previous findings from our laboratory of a deleterious role for di-18:0 diacylglycerol [18]. Other reports show a sixfold increase in C18:0 ceramide species in $o b / o b$ mice compared with lean controls [26], and a temporal relationship between C18:0 ceramide and the development of insulin resistance in skeletal muscle during high fat feeding in mice [27]. Thus, our data combined with the literature suggest a unique relationship between C18:0 muscle ceramide, obesity and insulin resistance.

Ceramide species containing stearate may be uniquely deleterious for insulin sensitivity by altering the biophysical properties of membranes. Ceramides form highly ordered domains in membranes, termed 'rafts', which are more readily formed by saturated species and are disrupted by ceramide species containing double bonds [28]. These rafts containing saturated ceramide would decrease membrane fluidity, which has been shown to influence insulin receptor function in the plasma membrane [29] and may alter protein function, which could conceivably promote mitochondrial dysfunction [30]. Moreover, C18:0 ceramide has been implicated in the activation of mitophagy [31], which is associated with muscle glucose metabolism [32] and hepatic insulin resistance [33]. Expression of the mitophagy marker gene MAPILC3A was higher in athletes, suggesting increased autophagosome 
Fig. 4 (a) Concentrations of muscle glucosylceramide species in the different groups at rest and (b) combined values for all groups at rest and during exercise and recovery. Percentage changes (c) from rest to exercise and (d) from rest to recovery. Values are means \pm SEM. White bars, obese individuals; light grey bars, type 2 diabetic patients; dark grey bars, athletes. *Significantly different from in athletes; ${ }^{\S}$ significantly different from at rest; " significantly different from during exercise ( $p<0.05$ for all). (e) Relationship between C18:0 glucosylceramide and insulin sensitivity. Circles, obese individuals; triangles, type 2 diabetic patients; squares, athletes $(r=-0.36, p=0.03)$. GlucCer, glucosylceramide $\mathbf{a}$

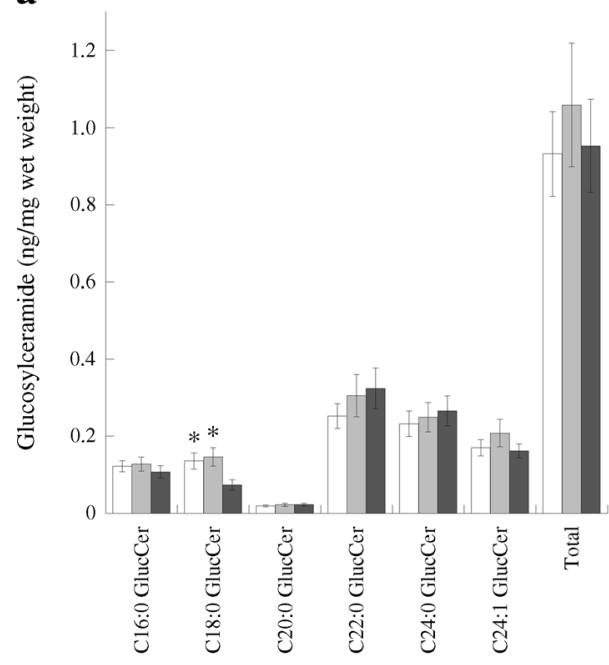

c
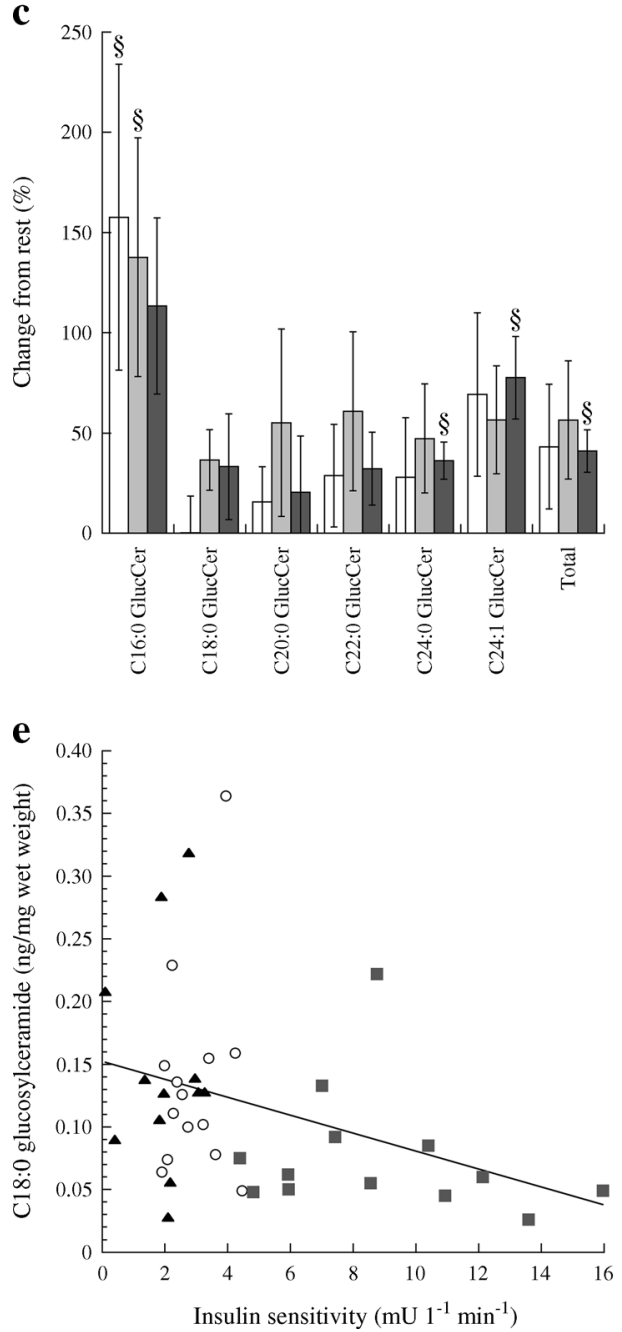

b

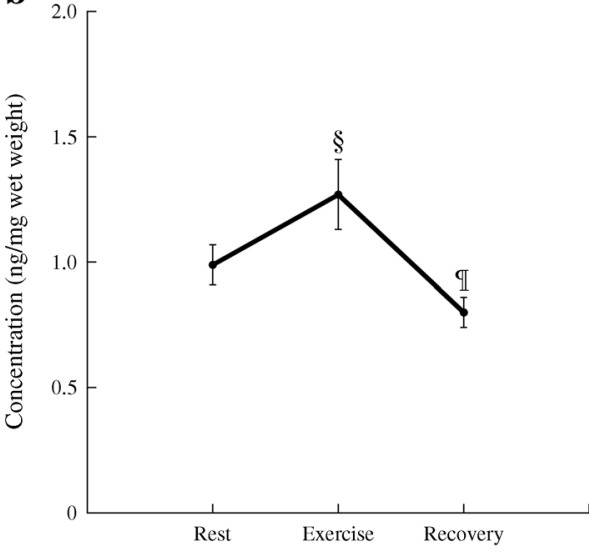

d

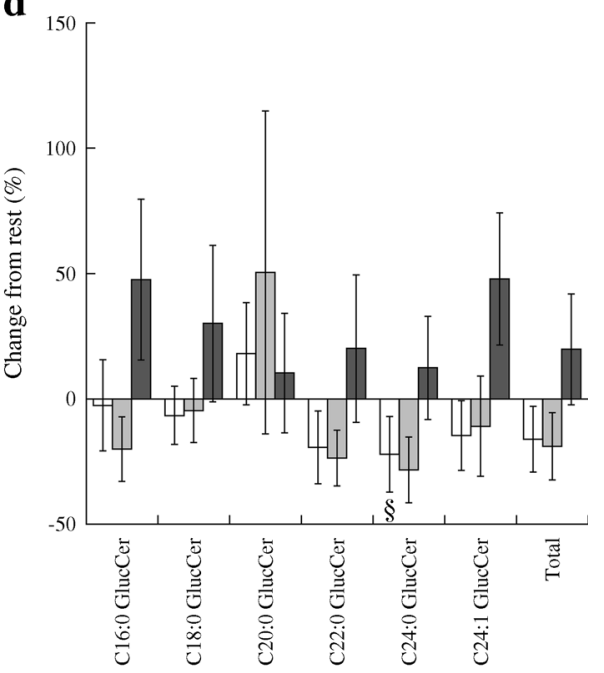

formation, but other markers were not different, suggesting similar activation of mitophagy among groups. Additionally, there were no relationships between mitophagy markers and C18:0 ceramide, suggesting that activation of mitophagy is not a likely explanation for the influence of C18:0 ceramide on insulin resistance. Finally, ceramide is known to activate protein phosphatase 2A (PP2A) to induce insulin resistance, and there is evidence suggesting that C18:0 ceramide 
Fig. 5 (a) Concentrations of muscle ganglioside species in the different groups at rest and (b) combined values for all groups at rest and during exercise and recovery. All values are means \pm SEM. White bars, obese individuals; light grey bars, type 2 diabetic patients; dark grey bars, athletes. *Significantly different from in athletes $(p<0.05)$ a

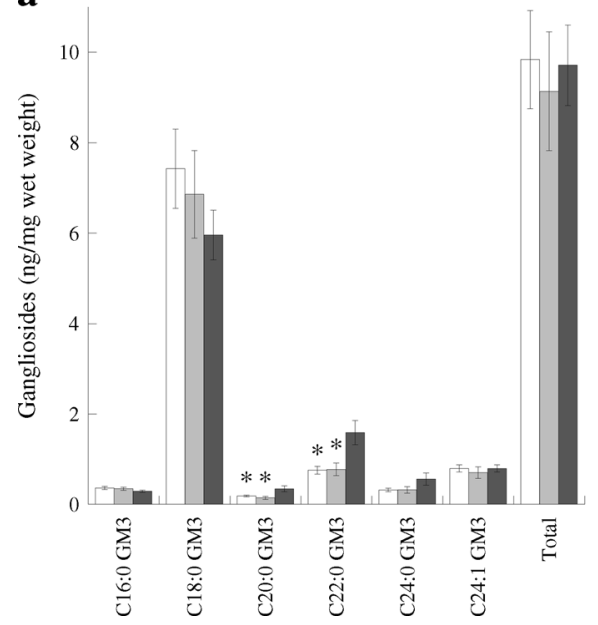

b

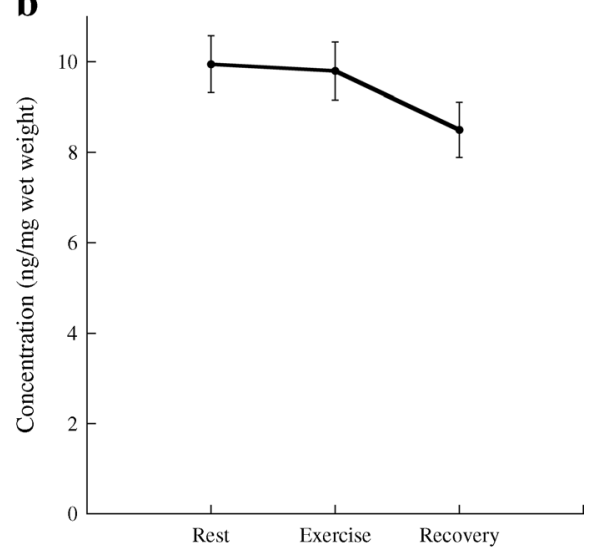

preferentially binds to the inhibitor of protein phosphatase $2 \mathrm{~A}$ (I2PP2A), thus preventing inhibition of PP2A [34]. Therefore, $\mathrm{C} 18: 0$ ceramide may promote insulin resistance by activating PP2A as well as altering the physical properties of membranes.

It is tempting to speculate that increased C18:0 ceramide, dihydroceramide and glucosylceramide species in insulinresistant skeletal muscle arise from the increased activity of ceramide synthase 1 (CERS1; encoded by CERS1). CERS1 is the most abundant ceramide synthase isoform and preferentially incorporates stearoyl-CoA [35]. Alterations in the muscle lipid supply may also influence ceramide species because type 2 diabetic patients had increased baseline serum C18:0 fatty acid content compared with obese individuals and athletes. Saturated serum NEFA may stimulate the synthesis of muscle ceramide and sphingolipids either directly or by the
Fig. 6 (a) Transcription levels of genes controlling ceramide production and clearance in the different groups at rest. Percentage changes in the combined values for all groups from (b) rest to exercise and (c) rest to recovery. mRNA expression was normalised to obese group levels and all values are means \pm SEM. White bars, obese individuals; light grey bars, type 2 diabetic patients; dark grey bars, athletes. ${ }^{\dagger}$ significantly different from in type 2 diabetic patients; ${ }^{*}$ significantly different from in obese individuals, $\S_{\text {significantly different from at }}$ rest ( $p<0.05$ for all). $A C E R$ encodes alkaline ceramidase; $A S A H$, acidic ceramidase; CERS, ceramide synthase; $D E G S$, dihydroceramide desaturase; $N E U$, sialidase; $S G M S$, sphingomyelin synthase; $S M P D$, acidic sphingomyelinase; $S P H K$, sphingosine kinase; SPTLC, serine palmitoyl-transferase; $U G C G$, glucosylceramide synthase
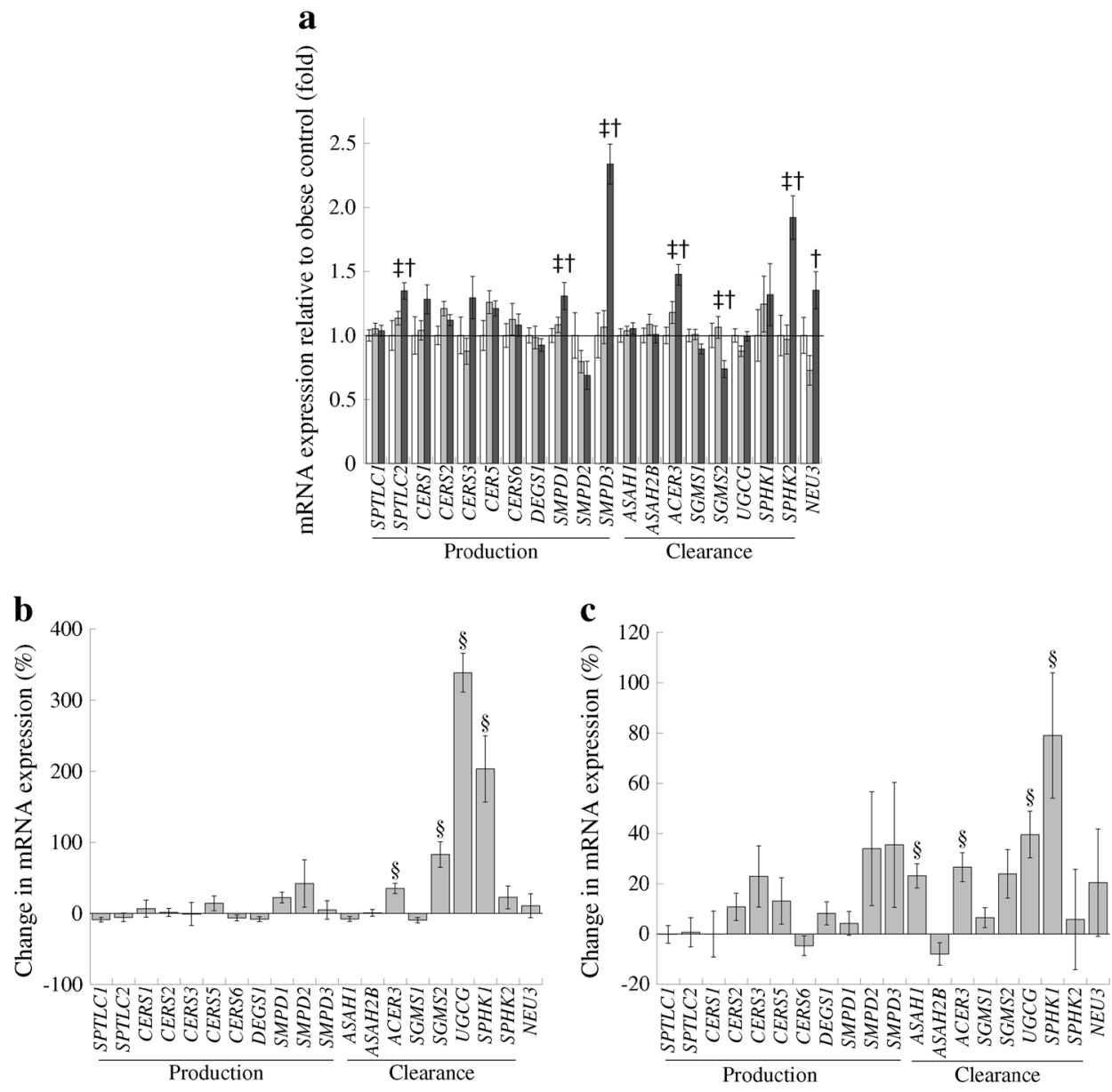
Fig. 7 Levels of skeletal muscle inflammatory markers in each group at rest, after exercise, and after recovery. Western blots and densitometry quantification showing levels of (a) JNK, (b) TLR4, (c) MAP4K4 and (d) ERK1/2. All values are means \pm SEM. White bars, obese individuals; light grey bars, type 2 diabetic patients (T2D); dark grey bars, athletes. ${ }^{\S}$ Significantly different from at rest; 'significantly different from type 2 diabetic patients; ${ }^{\ddagger}$ significantly different from obese individuals $(p<0.05$ for all). ERK1/2-P/T, phosphorylated/total ERK1/2; Ex, exercise; JNK-P/T, phosphorylated/total JNK; Rec, recovery $\mathbf{a}$

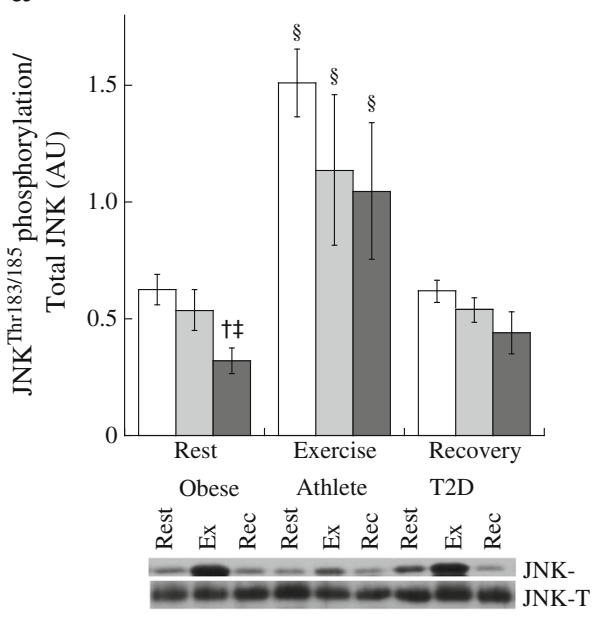

c

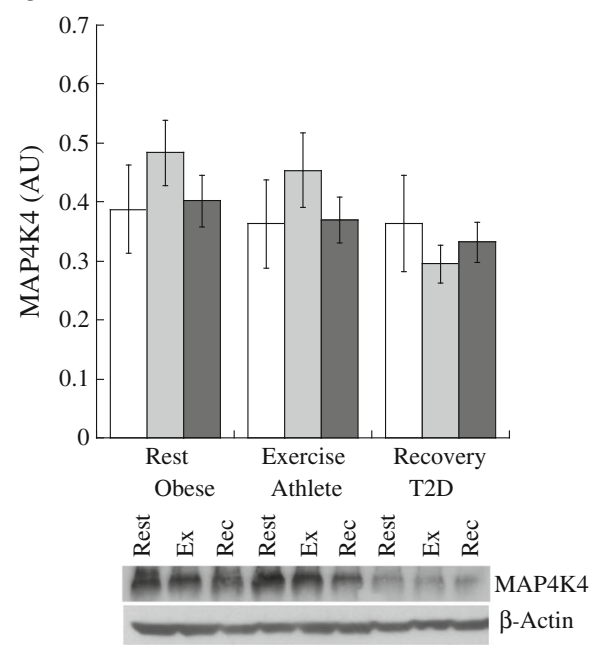

b

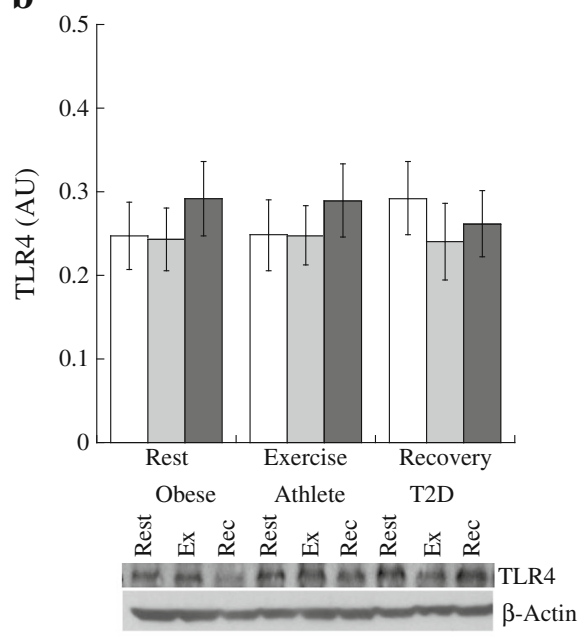

d

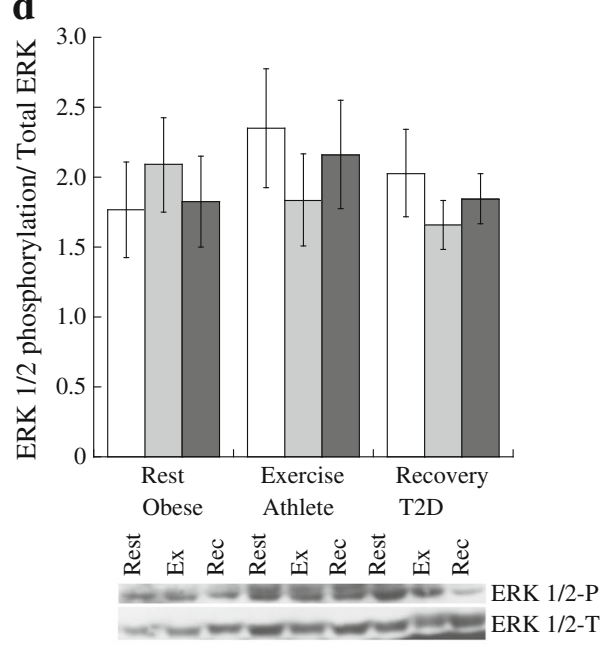

activation of TLR4 to promote de novo ceramide synthesis $[7,9]$. Our data are inconsistent with TLR4-induced activation of de novo ceramide synthesis, thus implicating muscle lipid supply in C18:0 ceramide accumulation. Increased muscle ceramide and sphingolipid synthesis may help to explain the link between dietary saturated fat, cardiovascular disease and diabetes $[36,37]$. Another potential explanation is greater sphingomyelinase activity in insulin-resistant states because the sphingomyelin composition of human muscle predominately contains stearate [14]. Increased sphingomyelinase activity generates ceramide and has been reported in insulinresistant liver [38] and in skeletal muscle from insulinresistant (vs insulin-sensitive) obese humans [39]. Further, inhibition of sphingomyelinase prevented saturated fat induced insulin resistance in skeletal muscle myotubes [40]. These data make sphingomyelinase a likely candidate to explain increased C18:0 ceramide species in skeletal muscle. Overall, our data revealed that muscle C18:0 ceramide, dihydroceramide and glucosylceramide species were related to muscle inflammation and insulin resistance, and may be increased by increased saturated fat consumption or increased sphingomyelinase activity. Therefore, reducing C18:0 ceramides and sphingolipids may be a particularly efficacious therapeutic aim to prevent and treat muscle insulin resistance.

Gene transcription data revealed that the capacity for ceramide and sphingolipid synthesis and degradation may be lower in obese and type 2 diabetic individuals than in athletes. If protein content follows gene expression, then these data are consistent with increased ceramide turnover in athletes compared with obese individuals with or without type 2 diabetes. Although we found no differences in ceramide content in this study, many studies have reported higher ceramide content in obese and insulin-resistant individuals compared with lean controls [4-9]. The gene transcription data presented here suggest that increased ceramide synthesis may not always be the cause, and indicates that decreased ceramide turnover may promote tissue accumulation.

Chronic endurance training decreases muscle ceramide content in rodents [41] and humans [10-12], although there 
are exceptions [42]. Additionally, endurance training decreased the $\mathrm{C} 18: 0$ sphingomyelin content of human muscle, which may help explain the lower C18:0 ceramide levels in athletes in this study [14]. However, the importance of a single session of exercise in changing muscle ceramide is unclear. It should be pointed out that the response to exercise may be species specific because muscle ceramide increased after a single exercise session in humans [14] but decreased in rodents $[15,43]$. Our muscle data agree with the only other study in humans: we found that exercise increased most muscle ceramides and other sphingolipid species. Of note, the exercise-induced increase in muscle C16:0 and C18:0 ceramide was exacerbated in obese and type 2 diabetic individuals compared with athletes, despite lower mRNA expression for ceramide synthesis genes. Recently, C16:0 muscle ceramide accumulation was found to inhibit anabolic signalling after resistance exercise in older humans [44]. Thus, the exaggerated increase in these ceramide species with exercise in obese and type 2 diabetic individuals may attenuate contraction-induced skeletal muscle adaptations and promote sarcopenia in insulin-resistant individuals.

Several mechanisms might explain the increase in muscle ceramide content immediately after exercise. Serum NEFA can activate TLR4 to promote cytokine and ceramide synthesis [45], implicating increased NEFA concentration during exercise. However, the NEFA concentration during exercise was unrelated to muscle ceramides and there were no changes in TLR4 or MAP4K4 content and IKK $\alpha$ or ERK1/2 phosphorylation. These data suggest that TLR4 activation does not explain the increase in muscle ceramide content during exercise. The most likely explanation is that the exercise-induced increase in muscle ceramide content is caused by the acute inflammatory response to exercise [46]. TNF- $\alpha$ concentration increased during exercise compared with at rest, coinciding with increased phosphorylation of JNK, a downstream target of TNF- $\alpha$ signalling. TNF- $\alpha$ rapidly increases ceramide content by increasing de novo ceramide synthesis, and this probably accounts for exerciseinduced ceramide accumulation [47].

After $2 \mathrm{~h}$ of recovery, most ceramides and other sphingolipids had decreased to basal levels, with sphingosine and ceramide content being significantly decreased compared with rest. Plasma TNF- $\alpha$ concentration and muscle JNK phosphorylation decreased during recovery compared with during exercise, suggesting that reduced post-exercise inflammatory signalling contributed to decreased muscle ceramide content, probably by decreasing de novo synthesis [47]. Additionally, exercise and recovery increased the expression of several genes responsible for ceramide clearance, including acidic and alkaline ceramidase 1 and 3, glucosylceramide synthase and sphingosine kinase 1, suggesting that increased ceramide degradation and clearance occurs after exercise (Fig. 6c). Therefore, decreased ceramide synthesis and increased clearance during recovery from acute exercise reduce muscle ceramide content and may help to explain the increased insulin sensitivity that results from a single exercise bout [48].

There are several limitations in this study that are important to address. There was no normal weight sedentary or normally physically active control group, which hinders interpretation of the effects of obesity and endurance exercise training on the outcomes measured. Because the post-exercise muscle biopsy was taken from the same site as the resting muscle biopsy, our exercise ceramide data may be confounded by muscle inflammation. We could not measure incorporation of the $\left[\mathrm{U}-{ }^{13} \mathrm{C}\right]$ palmitate tracer into ceramides to measure the rates of synthesis. Additionally, we do not have measures of insulin sensitivity after exercise to compare with changes in muscle ceramides.

In summary, muscle $\mathrm{C} 18: 0$ ceramide, dihydroceramide and glucosylceramide species were related to insulin resistance, suggesting they may be unique therapeutic targets for insulin sensitisation. Muscle ceramide and dihydroceramides were related to intramuscular inflammation, which may provide a mechanism to explain insulin resistance associated with these intracellular lipids. Transcription of sphingolipid synthesis genes was lower in type 2 diabetic patients, suggesting a decreased capacity for sphingolipid synthesis. Exercise increased the serum TNF- $\alpha$ content and muscle JNK phosphorylation, which are known to promote de novo ceramide synthesis and may explain the increase in muscle ceramide and other sphingolipid content. Two hours of recovery led to increased mRNA expression for ceramide clearance and degradation. Along with the decreased ceramide and sphingosine concentrations compared with the resting state, this finding may help explain insulin sensitisation after acute exercise.

Acknowledgements Some of these data were presented as an abstract at the ADA 75th Scientific Sessions in Boston, MA, USA, 5-9 June 2015.

Funding This work was partly supported by a National Institutes of Health General Clinical Research Center grant (no. RR-00036) and National Institute of Diabetes and Digestive and Kidney Diseases grants (nos KO1DK066219 and R01DK089170) to BCB.

Duality of interest statement The authors declare that there is no duality of interest associated with this manuscript.

Contribution statement $\mathrm{BCB}$ is the guarantor of this work. He had full access to all the study data and takes responsibility for the integrity of the data and the accuracy of the data analysis. BCB designed the study, tested participants, analysed the data, wrote the manuscript and approved the final version; JTB performed the lipidomics analysis, helped interpret this data, edited the manuscript and approved the final version; AS performed all western blots, analysed and interpreted these data, helped with participant testing, and edited and approved the final version of the manuscript; SB helped with participant testing and with data acquisition and analysis, 
and edited and approved the final version of the manuscript; AK tested participants, acquired and analysed data, and edited and approved the final version of the manuscript; HHB, PSa, PSi and MSK acquired, analysed and interpreted lipidomics data, and revised and approved the final manuscript; TW and MKT acquired, analysed and interpreted gene chip data, and revised and approved the final manuscript; LP helped design the study, provided medical oversight, performed all biopsies, and helped write and approved the final manuscript.

\section{References}

1. Coen PM, Goodpaster BH (2012) Role of intramyocelluar lipids in human health. Trends Endocrinol Metab: TEM 23:391-398

2. Samuel VT, Petersen KF, Shulman GI (2010) Lipid-induced insulin resistance: unravelling the mechanism. Lancet 375:2267-2277

3. Summers SA (2006) Ceramides in insulin resistance and lipotoxicity. Prog Lipid Res 45:42-72

4. Adams JM 2nd, Pratipanawatr T, Berria R et al (2004) Ceramide content is increased in skeletal muscle from obese insulin-resistant humans. Diabetes 53:25-31

5. Turinsky J, O'Sullivan DM, Bayly BP (1990) 1,2-Diacylglycerol and ceramide levels in insulin-resistant tissues of the rat in vivo. J Biol Chem 265:16880-16885

6. Moro C, Galgani JE, Luu L et al (2009) Influence of gender, obesity, and muscle lipase activity on intramyocellular lipids in sedentary individuals. J Clin Endocrinol Metab 94:3440-3447

7. Chavez JA, Summers SA (2003) Characterizing the effects of saturated fatty acids on insulin signaling and ceramide and diacylglycerol accumulation in 3T3-L1 adipocytes and $\mathrm{C} 2 \mathrm{C} 12$ myotubes. Arch Biochem Biophys 419:101-109

8. Blachnio-Zabielska A, Baranowski M, Zabielski P, Gorski J (2010) Effect of high fat diet enriched with unsaturated and diet rich in saturated fatty acids on sphingolipid metabolism in rat skeletal muscle. J Cell Physiol 225:786-791

9. Holland WL, Bikman BT, Wang LP et al (2011) Lipid-induced insulin resistance mediated by the proinflammatory receptor TLR4 requires saturated fatty acid-induced ceramide biosynthesis in mice. J Clin Invest 121:1858-1870

10. Bruce CR, Thrush AB, Mertz VA et al (2006) Endurance training in obese humans improves glucose tolerance and mitochondrial fatty acid oxidation and alters muscle lipid content. Am J Physiol Endocrinol Metab 291:E99-E107

11. Dube JJ, Amati F, Stefanovic-Racic M, Toledo FG, Sauers SE, Goodpaster BH (2008) Exercise-induced alterations in intramyocellular lipids and insulin resistance: the athlete's paradox revisited. Am J Physiol Endocrinol Metab 294:E882-E888

12. Dube JJ, Amati F, Toledo FG et al (2011) Effects of weight loss and exercise on insulin resistance, and intramyocellular triacylglycerol, diacylglycerol and ceramide. Diabetologia 54:1147-1156

13. Wojtaszewski JF, Hansen BF, Gade et al (2000) Insulin signaling and insulin sensitivity after exercise in human skeletal muscle. Diabetes 49:325-331

14. Helge JW, Dobrzyn A, Saltin B, Gorski J (2004) Exercise and training effects on ceramide metabolism in human skeletal muscle. Exp Physiol 89:119-127

15. Oakes ND, Bell KS, Furler SM et al (1997) Diet-induced muscle insulin resistance in rats is ameliorated by acute dietary lipid withdrawal or a single bout of exercise: parallel relationship between insulin stimulation of glucose uptake and suppression of long-chain fatty acyl-CoA. Diabetes 46:2022-2028
16. Bergman BC, Brozinick JT, Strauss A et al (2015) Serum sphingolipids: relationships to insulin sensitivity and changes with exercise in humans. Am J Physiol Endocrinol Metab 309:E398E408

17. Boston RC, Stefanovski D, Moate PJ, Sumner AE, Watanabe RM, Bergman RN (2003) MINMOD Millennium: a computer program to calculate glucose effectiveness and insulin sensitivity from the frequently sampled intravenous glucose tolerance test. Diabetes Technol Ther 5:1003-1015

18. Bergman BC, Hunerdosse DM, Kerege A, Playdon MC, Perreault L (2012) Localisation and composition of skeletal muscle diacylglycerol predicts insulin resistance in humans. Diabetologia 55: $1140-1150$

19. Guo Z, Mishra P, Macura S (2001) Sampling the intramyocellular triglycerides from skeletal muscle. J Lipid Res 42:1041-1048

20. Dalma-Weiszhausz DD, Warrington J, Tanimoto EY, Miyada CG (2006) The affymetrix GeneChip platform: an overview. Methods Enzymol 410:3-28

21. Straczkowski M, Kowalska I, Nikolajuk A et al (2004) Relationship between insulin sensitivity and sphingomyelin signaling pathway in human skeletal muscle. Diabetes 53:1215-1221

22. Thrush AB, Harasim E, Chabowski A, Gulli R, Stefanyk L, Dyck DJ (2011) A single prior bout of exercise protects against palmitateinduced insulin resistance despite an increase in total ceramide content. Am J Physiol Regul Integr Comp Physiol 300:R1200-R1208

23. Skovbro M, Baranowski M, Skov-Jensen C et al (2008) Human skeletal muscle ceramide content is not a major factor in muscle insulin sensitivity. Diabetologia 51:1253-1260

24. Hoy AJ, Brandon AE, Turner N et al (2009) Lipid and insulin infusion-induced skeletal muscle insulin resistance is likely due to metabolic feedback and not changes in IRS-1, Akt, or AS160 phosphorylation. Am J Physiol Endocrinol Metab 297:E67-E75

25. Coen PM, Dube JJ, Amati F et al (2010) Insulin resistance is associated with higher intramyocellular triglycerides in type I but not type II myocytes concomitant with higher ceramide content. Diabetes 59:80-88

26. Samad F, Hester KD, Yang G, Hannun YA, Bielawski J (2006) Altered adipose and plasma sphingolipid metabolism in obesity: a potential mechanism for cardiovascular and metabolic risk. Diabetes 55:2579-2587

27. Turner N, Kowalski GM, Leslie SJ et al (2013) Distinct patterns of tissue-specific lipid accumulation during the induction of insulin resistance in mice by high-fat feeding. Diabetologia 56:1638-1648

28. Pinto SN, Silva LC, Futerman AH, Prieto M (2011) Effect of ceramide structure on membrane biophysical properties: the role of acyl chain length and unsaturation. Biochim Biophys Acta 1808: 2753-2760

29. Storlien LH, Jenkins AB, Chisholm DJ, Pascoe WS, Khouri S, Kraegen EW (1991) Influence of dietary fat composition on development of insulin resistance in rats. Relationship to muscle triglyceride and omega- 3 fatty acids in muscle phospholipid. Diabetes 40 : 280-289

30. Grassme H, Riethmuller J, Gulbins E (2007) Biological aspects of ceramide-enriched membrane domains. Prog Lipid Res 46:161170

31. Sentelle RD, Senkal CE, Jiang W et al (2012) Ceramide targets autophagosomes to mitochondria and induces lethal mitophagy. Nat Chem Biol 8:831-838

32. He C, Bassik MC, Moresi V et al (2012) Exercise-induced BCL2regulated autophagy is required for muscle glucose homeostasis. Nature 481:511-515

33. Liu HY, Han J, Cao SY et al (2009) Hepatic autophagy is suppressed in the presence of insulin resistance and hyperinsulinemia: inhibition of FoxO1-dependent expression of key autophagy genes by insulin. J Biol Chem 284:31484-31492 
34. Mukhopadhyay A, Saddoughi SA, Song P et al (2009) Direct interaction between the inhibitor 2 and ceramide via sphingolipidprotein binding is involved in the regulation of protein phosphatase 2A activity and signaling. FASEB J 23:751-763

35. Laviad EL, Albee L, Pankova-Kholmyansky I et al (2008) Characterization of ceramide synthase 2: tissue distribution, substrate specificity, and inhibition by sphingosine 1-phosphate. J Biol Chem 283:5677-5684

36. Riserus U, Willett WC, Hu FB (2009) Dietary fats and prevention of type 2 diabetes. Prog Lipid Res 48:44-51

37. Dayton S, Pearce ML, Goldman H et al (1968) Controlled trial of a diet high in unsaturated fat for prevention of atherosclerotic complications. Lancet 2:1060-1062

38. Longato L, Tong M, Wands JR, de la Monte SM (2012) High fat diet induced hepatic steatosis and insulin resistance: role of dysregulated ceramide metabolism. Hepatol Res: Off J Jpn Soc Hepatol 42:412-427

39. Straczkowski M, Kowalska I, Baranowski M et al (2007) Increased skeletal muscle ceramide level in men at risk of developing type 2 diabetes. Diabetologia 50:2366-2373

40. Verma MK, Yateesh AN, Neelima K et al (2014) Inhibition of neutral sphingomyelinases in skeletal muscle attenuates fatty-acid induced defects in metabolism and stress. SpringerPlus 3:1-12

41. Dobrzyn A, Zendzian-Piotrowska M, Gorski J (2004) Effect of endurance training on the sphingomyelin-signalling pathway activity in the skeletal muscles of the rat. J Physiol Pharmacol 55:305313
42. Blachnio-Zabielska A, Zabielski P, Baranowski M, Gorski J (2011) Aerobic training in rats increases skeletal muscle sphingomyelinase and serine palmitoyltransferase activity, while decreasing ceramidase activity. Lipids 46:229-238

43. Dobrzyn A, Gorski J (2002) Ceramides and sphingomyelins in skeletal muscles of the rat: content and composition. Effect of prolonged exercise. Am J Physiol Endocrinol Metab 282:E277E285

44. Rivas DA, Morris EP, Haran PH et al (2012) Increased ceramide content and NFkappaB signaling may contribute to the attenuation of anabolic signaling after resistance exercise in aged males. J Appl Physiol 113:1727-1736

45. Shi H, Kokoeva MV, Inouye K, Tzameli I, Yin H, Flier JS (2006) TLR4 links innate immunity and fatty acid-induced insulin resistance. J Clin Invest 116:3015-3025

46. Ostrowski K, Rohde T, Asp S, Schjerling P, Pedersen BK (1999) Pro-and anti-inflammatory cytokine balance in strenuous exercise in humans. J Physiol 515(Pt 1):287-291

47. Strle K, Broussard SR, McCusker RH et al (2004) Proinflammatory cytokine impairment of insulin-like growth factor I-induced protein synthesis in skeletal muscle myoblasts requires ceramide. Endocrinology 145:4592-4602

48. Newsom SA, Everett AC, Hinko A, Horowitz JF (2013) A single session of low-intensity exercise is sufficient to enhance insulin sensitivity into the next day in obese adults. Diabetes Care 36: 2516-2522 